\title{
A novel methodology for optimum energy consumption in wireless sensor networks
}

\author{
Mohamed Abbas and Nasser Otayf \\ Electrical Engineering Department, College of Engineering, King Khalid University, \\ Abha, Saudi Arabia
}

\begin{abstract}
Purpose - The purpose of this paper is to minimize energy usage by maximizing network life in the creation of applications and protocols

Design/methodology/approach - This paper presents a novel methodology for optimum energy consumption in wireless sensor networks. The proposed methodology introduces some protocols and logarithms that effectively contributed to reducing energy consumption in these types of networks.

Findings - The results of that comparison showed the ability of those logarithms and protocols to reduce that energy but in varying proportions. It can be concluded that a significant reduction in energy consumption approximately $50 \%$ could be obtained by the proposed methodology.

Originality/value - Here, a novel methodology for optimum energy consumption in wireless sensor networks has been introduced.
\end{abstract}

Keywords Algorithms, Energy consumption, Efficient clustering, Protocols, WSN

Paper type Research paper

\section{Introduction}

Most studies have established different approaches, algorithms, clusters and protocols to archive maximum energy efficiency on energy consumption in wireless sensor technology. Wirless sensor network (WSN) consists of several nodes used for multi-hopping communication. To apply physical and environmental monitoring conditions such as temperature, sharpness, vibes, pressure, movement and pollutants, WSNs may be defined as self-configuring, wireless networks free of all infrastructure, which track or transmit data through the network to a central point or sink. The user-to-network interface is the baseline or sink. You can collect the relevant network information by injecting queries and collecting sink data. In general, a wireless network has thousands of nodes. The wireless node includes the modules, transceivers and components of electricity. Any node within the WSN limits the speed of operation, store and bandwidth. The first module concerns the active area sensing while the second involves nodes that are in sleep mode to conserve the energy presented in Srbinovska and Cundeva-Blajer (2019). Genetic algorithm (GA) can be deployed in various ways through modifications to the strategy being used. Another application of GA is seen in Panhwar et al. (2018). The proposal involves improved fitness functions of a GA in enhancing the distance-based energy optimization in WSNs. The authors proposed a GA-based optimization in the stationary WSNs with the deployment of multiple sinks. A modified GA that minimizes energy consumption in WSNs through an energy balance filter was proposed in Alisa and Hussein (2016). The proposed strategy involves an intelligent clustering protocol

(C) Mohamed Abbas and Nasser Otayf. Published in Frontiers in Engineering and Built Environment. Published by Emerald Publishing Limited. This article is published under the Creative Commons Attribution (CCBY 4.0) licence. Anyone may reproduce, distribute, translate and create derivative works of this article (for both commercial and non-commercial purposes), subject to full attribution to the original publication and authors. The full terms of this licence may be seen at http://creativecommons. org/licences/by/4.0/legalcode
Received 6 March 2021 Revised 28 March 2021 Accepted 31 March 2021 
FEBE 1,1

\section{6}

Figure 1.

The infrastructure of wireless sensor network that aims at prolonging the network lifetime and minimizing energy consumption. For the protocol to perform clustering with a dynamic number of clusters, it requires a proposed modified GA that conducts the process of selecting the optimum number of clusters and electing the suitable cluster heads. The modified GA target is to ensure the energy consumed by nodes in the round is minimized. Figure 1 shows the infrastructure of a WSN. Often, after deploying sensor nodes, they are responsible for powerful multi-hop network infrastructure.

In the energy-efficient $\beta$-approximate skylines processing in WSNs, the energy-efficient processing algorithm using mapping and filtering strategies, named Actual Approximate Skyline (AAS), was proposed in Xin et al. (2015). And more than that, an extended algorithm named Hypothetical Approximate Skyline (HAS), which replaces the real tuples with the hypothetical ones, is proposed to further reduce the communication cost. The AAS algorithm helps in reducing the communication cost of evaluating the $\beta$-approximate skyline. The study indicates that both the AAS and HAS are energy-efficient in evaluating approximate skylines in WSNs. The Bat algorithm is proposed in Sangaiah et al. (2019), which is used in selecting the optimum monitoring sensor node and the appropriate path that can be used to reduce energy consumption. The simulation results of the proposed algorithm show that the proposed algorithm has been able to reduce the power consumption of the network and increase the lifetime of the network. In the study by Baba and Rao (2016), the integration of progressive sleep scheduling (PSS) algorithm with opportunistic routing protocol is proposed to improve the network lifetime of a WSN. The algorithm provided by the PSS enables the selection of the best relay nodes in the network by the opportunities routing protocol and enables the mode sleep to be applied when the relay node is completed. The changing of mode is achieved by applying the PSS algorithm. In this algorithm, some nodes are working, and the remaining nodes are in sleep mode; the working nodes are selected by the protocol mechanism is the best path selection of the nodes. Various schemes have been developed concerning optimum energy conception in WSNs. The improved harmony search algorithm was implemented in a pre-defined scheme that involved node deployment strategy with modification in equations where the range number of selecting the random number increases in Halim et al. (2018). The location of the sink nodes is also changed to find the ideal location and reduce the nodes' energy consumption. The results of both experiments give a positive reaction where the energy consumption decreases as the range number increases. A new energy balance model utilizes a shuffled frog leaping algorithm. In the model, the mathematical expression of energy consumption in the physical layer is given with transmit power at first, received power and signal bandwidth, and the objective optimization function of energy consumption balance is built by the total sending energy consumption and transmission power of WSN proposed in Zhou et al. (2018). Another scheme built to achieve the energy consumption balance is the novel grid-based hybrid

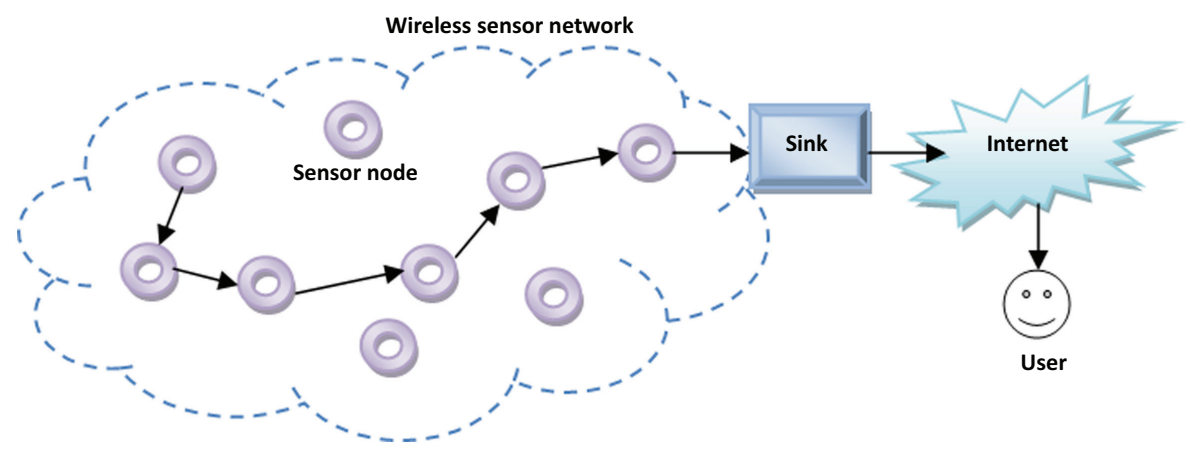


network deployment framework, which ensures energy efficiency and load balancing in WSNs. The fuzzy-based solution for data fusion in WSNs is proposed, which provides a better quality of service (QoS) and reduces energy consumption in Collotta et al. (2017). An energy-efficient broadcasting scheme was proposed for smart industrial WSNs in Chen et al. (2015). The scheme incorporates an adjustable broadcasting radius that improves the scheme's ability to improve network upgrades. Using the TinyMD algorithm, a WSN power saver diagram can transform sensor data and this methodology is presented in detail in Bok et al. (2016). The dual-mode Interest forwarding (DMIF) scheme is proposed in Gao et al. (2016) for named data networking (NDN)-based WSNs. The aim of this paper is to propose a methodology to reduce energy consumption compared to the current model after deployment in the sensor nodes and transmits at a lower energy level. The next sections present the proposed methodology to reduce the energy consumption in WSNs and results in discussion.

\section{Proposed methodology to reduce energy consumption in WSNs}

Energy optimization is an essential issue in the study of WSNs. It is important to minimize the consumption of energy or power in the network nodes deployed on the WSN to maximize the lifetime of the nodes. This study explores the performance of the current WSN in this project and proposes ways to optimize the energy consumption using the key parameters of the energy models in WSN. This study gives a comparative analysis of the performance of the present model and the proposed energy models. Finally, the proposed model demonstrates reduced energy consumption compared to the current model after deployment in the sensor nodes and transmits at a lower energy level. The optimization of energy in the WSN is done by the actions given in the following:

(1) Minimizing the distance separating the cluster heads as well as mobile sinks;

(2) Maximizing the number of active nodes;

(3) Minimizing the number of dead nodes;

(4) Retaining the amount or energy input;

(5) Minimizing the distance between the sensors.

\subsection{Proposed optimization procedures}

Due to the need to save as much energy as possible in each sensor node, energy conservation is a critical component of WSNs. Energy is the primary bottleneck in sensor node communication, and most of the available energy in a sensor node is used to communicate. Data transmission uses the same amount of energy whether the sender and receiver are near each other or are situated a large distance apart (between the nodes). To optimize communications, it is imperative that the distance between the nodes with near proximity to the base station and the nodes at the far end of the long-distance connection be reduced. This is another one of the algorithms that is used in this example, and it is used to create clusters of sensor nodes. A single cluster head is randomly chosen from all the nodes in the cluster. The clustering that happens when many nodes come together to form cluster-heads decreases internode distances, and network energy consumption often decreases as a result. The second technique for optimizing energy usage is to find dead nodes and turn them off. They are no longer connected, use no energy, and do not have any impact on the overall energy consumption. To keep the energy losses in the dead nodes to a minimum, the number of dead nodes must be decreased. Optimization demands that the three parameters be specified and defined precisely. This allowed the number of dead nodes in this scenario to be reduced from 2
Energy consumption in wireless sensor network 
FEBE 1,1

\section{8}

to 1 . When all the values for the energy inputs and energy outputs had been fixed, the final step was to constrain the value of the energy input, to avoid excessive energy supply that can lead to wasteful usage of energy. In the following graphic, you can see how optimization of energy consumption was done using the following steps:

Let us begin with phase one. If you want to do this, you will need to have a significant increase in the number of nodes (from 100 to 200 or more).

The programme for energy efficiency starts by increasing the number of live nodes to the maximum possible to optimize cluster sizes and to minimize inter-node distance. Go to Step 2 to find out how far apart the nodes are when doubled: Find out how far apart the nodes are when doubled, and then reduce the distance between the nodes to a maximum of 4,999. In step 3 , dead nodes are reduced to the maximum extent possible to release the most energy to the live nodes. In the final stage, you must keep track of the input energy of energy for data aggregation (EDA), which is 9 Joules, using the formulae mentioned below. ( 5 multiplied by 10 increased to the power of-9) manufacturing and construction, the following flowchart is shown in Figure 2, which explains the optimization process described above.

\section{Results and discussion}

This section explores how the solution presented has proven to be effective, as the results that have been shown to find out if a proposed solution has been implemented. The number of operating nodes will increase by a factor of two. The nodes commonly used in implementing an application (such as application servers or application programming interface (API) servers) and operational nodes can appear identical in the early stages of implementation, but as the implementation progresses, these may move dramatically (which may be application servers or API servers). Even if it could double the number of nodes every time a new one is added, working to extend the network by this factor would be

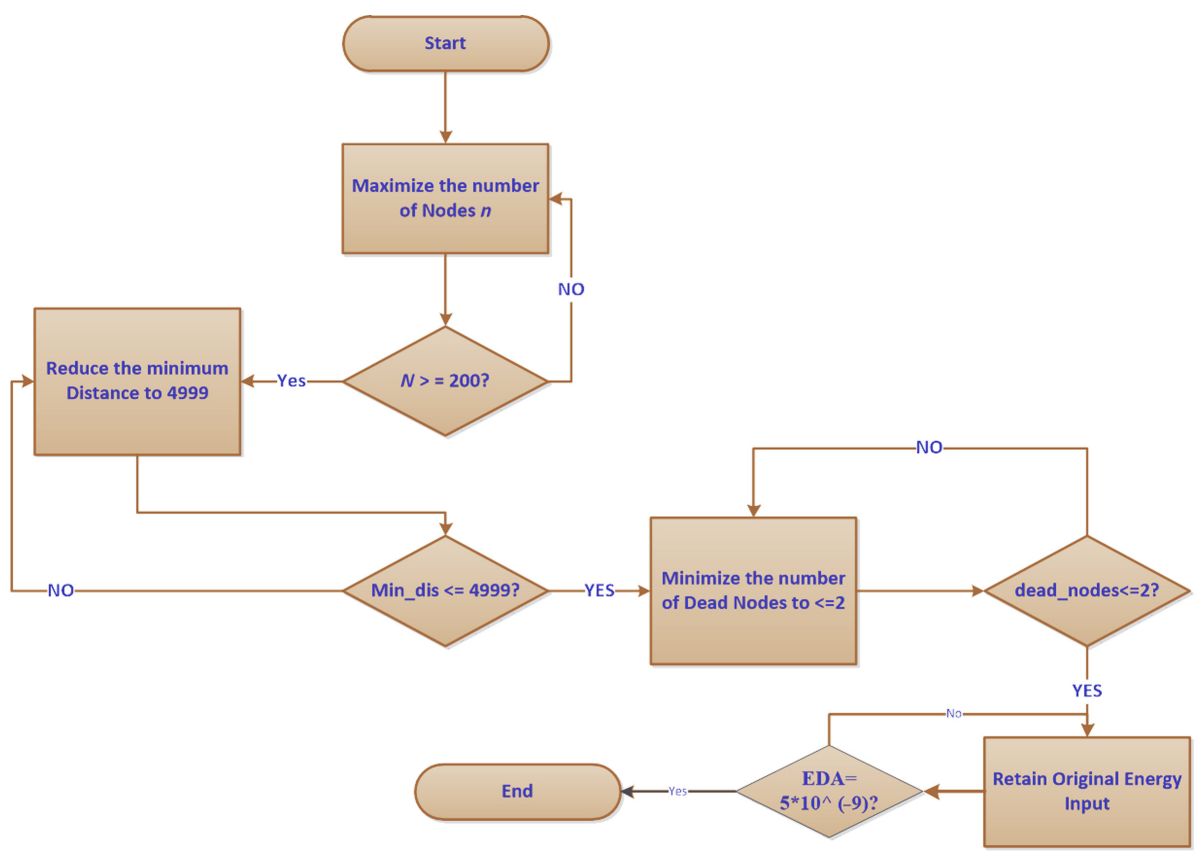

Figure 2.

Flowchart of the proposed methodology 
extremely challenging from the start, because the network could have less than 50 active nodes at that time. By looking at Figure 3, it is shown that the results show how it operates by comparing Figure 3 . These are examples of operating points discovered in the technique, but Figure 4 shows the broad range of locations where they have been found across a wide range of scales. The world was recognizing digital television as a big waste of time and bandwidth as an additional encumbrance and getting in the way of information transfer. SimpleNet has already engaged in extensive research on several conventional means to reduce energy consumption, as evidenced in the two examples shown in Figure 5. As shown in these figures, it is clearly demonstrated that current reliance on visualization has created a space between the proposed solution and the researchers and the data, and the strategy. It has been demonstrated that the proposed methodology reduces energy consumption by half of the conventional process. WSN specific tests show that our hope of a possible decrease in overall network energy consumption is warranted if long as the solution works, as evidenced by these, we see in line.

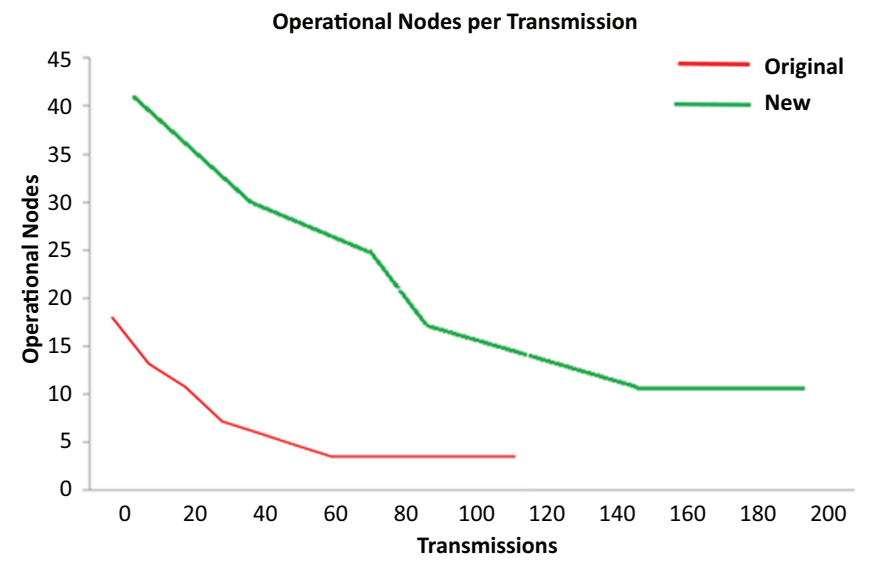

Figure 3.

Conventional operational nodes vs operational nodes in proposed methodology

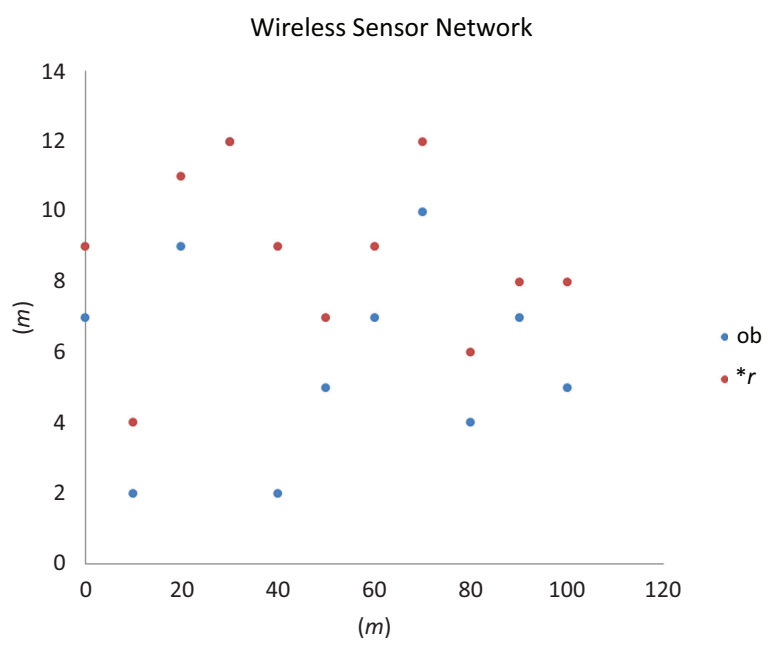

Figure 4.

Distribution of proposed nodes of WSN 


\section{FEBE 1,1}

\section{Figure 5.}

Conventional energy consumption vs. energy consumption in proposed methodology

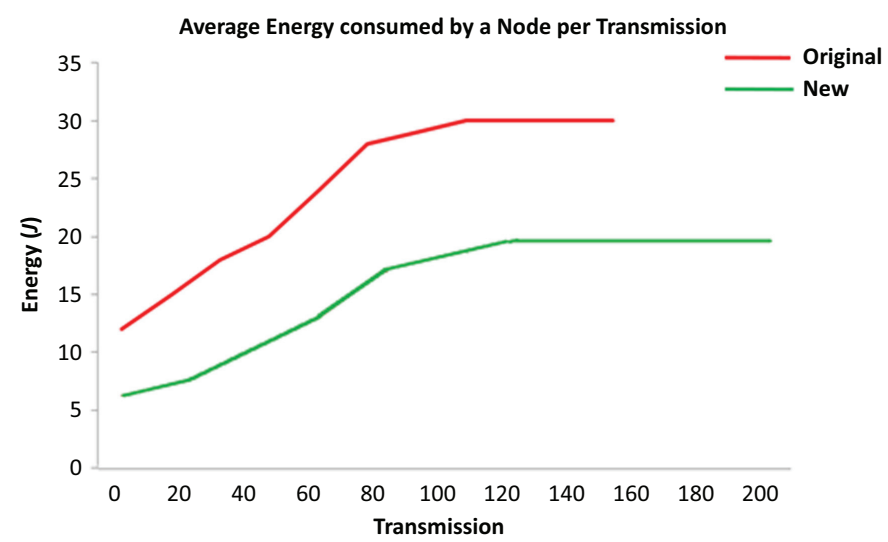

\section{Conclusion}

Most power is consumed in communications when sensor nodes transmit or receive information. During transmission or receiving processes, it is important to have energy-efficient devices to minimize the overall energy consumption. A novel methodology has been used to set the objective function to minimize the total energy consumption of the WSN under a given threshold. The aim of this paper is to establish a novel methodology for optimum energy consumption in WSNs. This suggested approach involves protocols and algorithms that have a notable influence on the reduction of energy consumption in these kinds of networks. The test compared the effects of algorithms and protocols, and the findings show that certain algorithms and protocols have the potential to minimize the energy by a certain amount, but it varies from case to case. It is plausible to assume that if we use the proposed approach, a reduction in energy consumption.

\section{References}

Alisa, Z.T. and Nassrullah, H.A. (2016), "Minimizing energy consumption in wireless sensor networks using modified genetic algorithm and an energy balance filter", 2016 Al-Sadeq International Conference on Multidisciplinary in IT and Communication Science and Applications (AICMITCSA), pp. 1-6, doi: 10.1109/AIC-MITCSA.2016.7759947.

Baba, S.B. and Rao, K.R.M. (2016), "Improving the network life time of a wireless sensor network using the integration of progressive sleep scheduling algorithm with opportunistic routing protocol", Indian Journal of Science and Technology, Vol. 9 No. 17, doi: 10.17485/ijst/2016/ v9i17/93011.

Bok, K., Lee, Y.J., Park, J. and Yoo, J. (2016), "An energy-efficient secure scheme in wireless sensor networks", Journal of Sensors, No. 2016, pp. 1321079-11321079, doi: 10.1155/2016/1321079.

Chen, J.J., Jia, J., Dai, E., Wen, Y. and Zhao, D. (2015), "Bicriteria optimization in wireless sensor networks: link scheduling and energy consumption", Journal of Sensors, Vol. 2015, pp. 724628-1724628, doi: 10.1155/2015/724628.

Collotta, M., Pau, G. and Bobovich, A.V. (2017), "A fuzzy data fusion solution to enhance the QoS and the energy consumption in wireless sensor networks", Wireless Communications and Mobile Computing, Vol. 2017, doi: 10.1155/2017/3418284.

Gao, S., Zhang, H. and Zhang, B. (2016), "Energy efficient interest forwarding in NDN-based wireless sensor networks", Mobile Information Systems Vol. 2016, pp. 3127029-13127029, doi: 10.1155/ 2016/3127029. 
Halim, N.H., Isa, A., Hamid, A., Isa, I., Mahyuddin, M., Saat, S. and Zin, M. (2018), "A pre-defined scheme for optimum energy consumption in wireless sensor network", Journal of Telecommunication, Electronic and Computer Engineering, Vol. 10, pp. 145-148.

Panhwar, M.A., Deng, Z., Khuhro, S.A. and Hakro, D. (2018), "Distance based energy optimization through improved fitness function of genetic algorithm in wireless sensor network", Studies in Informatics and Control, Vol. 27, pp. 461-468, doi: 10.24846/v27i4y201810.

Sangaiah, A.K., Sadeghilalimi, M., Hosseinabadi, A.R. and Zhang, W. (2019), "Energy consumption in point-coverage wireless sensor networks via Bat algorithm", IEEE Access, Vol. 7, pp. 180258-180269, doi: 10.1109/ACCESS.2019.2952644.

Srbinovska, M. and Cundeva-Blajer, M. (2019), "Optimization methods for energy consumption estimation in wireless sensor networks", Journal of Sustainable Development of Energy, Water and Environment Systems, Vol. 7, doi: 10.13044/j.sdewes.d6.0244.

Xin, J., Wang, Z., Bai, M., Ding, L. and Wang, G. (2015), "Energy-efficient $\beta$-approximate skylines processing in wireless sensor networks", Mathematical Problems in Engineering, Vol. 2015, pp. 1-11, doi: 10.1155/2015/149513.

Zhou, C., Wang, M., Qu, W. and Lu, Z. (2018), "A wireless sensor network model considering energy consumption balance”, Mathematical Problems in Engineering, Vol. 2018, pp. 1-8, doi: 10.1155/ $2018 / 8592821$.

\section{Corresponding author}

Mohamed Abbas can be contacted at: mabas@kku.edu.sa

For instructions on how to order reprints of this article, please visit our website:

www.emeraldgrouppublishing.com/licensing/reprints.htm

Or contact us for further details: permissions@emeraldinsight.com 